\title{
Involvement of Kruppel-like factor 9 in bleomycin-induced pulmonary toxicity
}

\author{
YUE GU $^{1}$, YAN-BING WU ${ }^{2}$, LI-HUI WANG ${ }^{1}$ and JIA-NING YIN $^{3}$ \\ ${ }^{1}$ Department of Respiratory and Critical Care Medicine, The First Hospital of Jilin University, Changchun, Jilin 130021; \\ ${ }^{2}$ Department of Respiratory and Critical Care Medicine, Beijing Chao-Yang Hospital Affiliated to Capital Medical University, \\ Beijing 100020; ${ }^{3}$ Department of Pediatrics, The First Hospital of Jilin University, Changchun, Jilin 130021, P.R. China
}

Received September 2, 2014; Accepted May 15, 2015

DOI: $10.3892 / \mathrm{mmr} .2015 .4015$

\begin{abstract}
Oxidative stress or the production of reactive oxygen species (ROS) has been implicated as an important factor in the development of bleomycin (BLM)-induced pulmonary toxicity; however, the mechanism behind the toxicity remains to be elucidated. The present study aimed to investigate the key factor involved in BLM-induced toxicity. The study was conducted in human pulmonary fibroblast (HPF) cells and in a mouse model. The ROS level, cell death assay, protein and gene expression levels of Kruppel-like factor 9 (Klf9) and other associated factors were assessed. A dose-dependent increase in ROS, lipid peroxidation, cell death, and protein and mRNA expression levels of NF-E2-related transcription factor 2 (Nrf2) and Klf9 were observed in BLM-treated cells. However, the expression levels of the other antioxidant proteins assessed, including catalase, super oxide dismutase, glutathione reductase and thioredoxin reductase 2 , were decreased. The expression levels of Nrf2 were decreased in cells treated with a higher concentration $(>200 \mu \mathrm{M})$ of BLM. These results suggested that in response to increased intracellular levels of ROS, above a critical threshold, Nrf2 stimulates the expression of Klf9, resulting in a further increase in Klf9-mediated ROS production and subsequent cell death. Furthermore, the data suggested that Klf9 may be considered as an adjunctive therapeutic target for BLM-induced pulmonary toxicity.
\end{abstract}

\section{Introduction}

Previous studies have suggested that alteration of oxidative stress and thiol-redox are initiating and propagating forces in the pathogenesis of bleomycin (BLM)-induced pulmonary

Correspondence to: Dr Jia-Ning Yin, Department of Pediatrics, The First Hospital of Jilin University, 71 Xinmin Street, Changchun, Jilin 130021, P.R. China

E-mail: yinjianing71@hotmail.com

Key words: human pulmonary fibroblast, Kruppel-like factor 9 knockout mice, NF-E2-related transcription factor 2, oxidative stress fibrosis $(1,2)$. BLM is an effective chemotherapeutic agent used primarily in the treatment of lymphoma, squamous cell carcinoma, testicular cancer and malignant pleural effusion (3). The antineoplastic effect of BLM is hypothesized to be due to the formation of a BLM-oxygen complex, which binds to the DNA and cleaves the phosphodiester-deoxyribose backbone (4). However, the effective clinical use of BLM in chemotherapy is limited as a result of the development of dose- and time-dependent interstitial pneumonitis, which eventually progresses to interstitial pulmonary fibrosis $(5,6)$.

Oxidative stress in the cell is caused by an imbalance between the formation of free radicals and their removal by enzymatic and non-enzymatic anti-oxidant molecules (7). It was hypothesized that redox cycling of the iron-BLM complex catalyzes the formation of reactive oxygen species (ROS), which leads to an imbalance between ROS and the available anti-oxidant defenses, principally glutathione (8). The generated ROS damage lung cells and activate inflammatory cells, which accumulate in the lower airways and produce further ROS, leading to oxidative stress. The nuclear transcription factor, NF-E2-related transcription factor $2(\mathrm{Nrf} 2)$, is a major sensor of oxidative stress in the cell (9). Under oxidative stress, Nrf2 is phosphorylated and translocated into the nucleus, where it activates the transcription of anti-oxidant and detoxifying genes by binding to the anti-oxidant response elements (AREs) in their regulatory regions (9).

A previous study investigating Kruppel-like factor 9 (Klf9) genes binding to the sequences of transcription factors in order to regulate the oxidative stress response, suggested that Klf9 promoter regions contained several conserved AREs (10), which are binding sites for $\mathrm{Nrf2}$, a major regulator of anti-oxidant defense in the cell $(9,11)$. Klf9 is a ubiquitously expressed member of the Sp1 C2H2-type zinc finger family of transcription factors (12). The expression of Klf9 can be increased by several stress-inducing agents, including the proteasomal inhibitor, bortezomib, and the histone deacetylase inhibitor, panobinostat, and Klf9 in turn mediates their cytotoxicity (13). Therefore, the present study aimed to investigate whether Klf9 was involved in the pathogenesis of BLM-induced pulmonary toxicity in human pulmonary fibroblasts (HPF), which was also analyzed in the Klf9 knockout mouse model. 


\section{Materials and methods}

Cell lines and reagents. HPF cells, isolated from human lung tissue, were obtained from Promocell (Heidelberg, Germany). The cells were cultured in Dulbecco's modified Eagle's medium (Sigma-Aldrich, St. Louis, MO, USA), supplemented with $10 \%$ fetal calf serum (Gibco-BRL, Gaithersburg, MD, USA), $2 \mathrm{mM}$ glutamine (Gibco-BRL), and penicillin-streptomycin antibiotics (Gibco-BRL). Hydrogen peroxide, sulforaphane, BLM, 2'-7'-dichlorofluorescin diacetate (DCFH-DA), actinomycin D and cycloheximide were purchased from Sigma-Aldrich.

Determination of cell viability and measurement of ROS. The HPF cells were seeded in a 96-well tissue culture plate, at a density of $\sim 1.25 \times 10^{4}$ cells/well, for $24 \mathrm{~h}$. The media was discarded and the cells were treated with various concentrations $(25,50,100$ or $200 \mu \mathrm{M})$ of BLM in serum-free media for $24 \mathrm{~h}$. The levels of intracellular ROS generation were measured using a well-characterized probe, DCFH-DA. DCFH-DA is hydrolyzed by esterases to DCFH, which is trapped within the cell. This non-fluorescent molecule is subsequently oxidized to fluorescent DCF by the action of cellular oxidants. The cells treated with either BLM or standard media for $6 \mathrm{~h}$ were subjected to DCFH staining and the fluorescence was determined at $485 \mathrm{~nm}$ excitation and $520 \mathrm{~nm}$ emission, using a microplate reader (FLUOstar; BMG Labtech, Inc., Durham, $\mathrm{NC}, \mathrm{USA})$.

Protein expression levels of antioxidants. The harvested control and BLM-treated (50 or $100 \mu \mathrm{M})$ HPF cells were washed with ice-cold phosphate-buffered saline and were lysed with radioimmunoprecipitation buffer (Cell Signaling Technology, Inc., Danvers, MA, USA). The lysates were centrifuged at $10,000 \mathrm{x}$ for $10 \mathrm{~min}$ at $4^{\circ} \mathrm{C}$, and the protein content of the supernatant was determined by the Bradford method (14). The proteins (10-20 $\mu \mathrm{g})$ were loaded and separated by $10 \%$ SDS-PAGE and were blotted onto a polyvinylidene fluoride membrane (Thermo Fisher Scientific, Inc., Frederick, MD, USA). The membranes were blocked with 5\% non-fat dry milk powder solution for $1 \mathrm{~h}$ at room temperature prior to overnight incubation with the following primary antibodies: Catalase (CAT; rabbit polyclonal; cat. no. ab87529; 1:500), glutathione reductase (GR; rabbit polyclonal; cat. no. ab137513; 1:500), super oxide dismutase (SOD; rabbit polyclonal; cat. no. ab16831; 1:2,000) and thioredoxin reductase 2 (TR-2; rabbit polyclonal; cat. no. ab71262; 1:500), all purchased from Abcam (Cambridge, MA, USA), and Nrf2 (rabbit polyclonal; cat. no. sc-722; 1:500) and Klf9 (rabbit polyclonal; cat. no. sc-28195; 1:500) were purchased from Santa Cruz Biotechnology, Inc. (Santa Cruz, CA, USA) at $4^{\circ} \mathrm{C}$. Following rinsing of the membranes with Tris-buffered saline with Tween ${ }^{\circledR} 20$, (Sigma-Aldrich), they were incubated with secondary antibody (goat anti-rabbit; polyclonal; cat. no. ab97200; 1:2,000; Abcam) for $1 \mathrm{~h}$ at room temperature and the bands were made visible by enhanced chemiluminescence using the SuperSignal West Femto substrate (Thermo Fisher Scientific, Inc.), visualized using the Alpha Innotech FluorChem HD2 Imaging system (Alpha Innotech Co., San Leandro, CA, USA). All antibodies, with the exception of $\alpha$-tubulin (rabbit polyclonal; cat. no. ab4074; 1:1,000; Abcam), were used at a dilution of 1:250, and the $\alpha$-tubulin antibody was used at a dilution of 1:1,000.

Gene expression analysis. Reverse transcription-quantitative polymerase chain reaction (RT-qPCR) was performed to analyze the gene expression status using gene-specific primers with the following sequences: Forward: 5'-ACAGTGGCT GTGGGAAAGTC-3' and reverse: 5'-AACTGCTTTTCCCCA GTGTG-3' for KLF9; and forward: 5'-AGTGGATCTGCC AACTACTC-3' and reverse: 5'-CATCTACAAACGGGAATG TCTG-3' for Nrf2. The total RNA was extracted from retinal cells using the RNA Nanoprep kit (Agilent Technologies, Santa Clara, CA, USA) and was reverse transcribed using the Reverse Transcription system (Promega Corporation, Madison, WI, USA) to synthesize cDNA. RT-qPCR was performed on the Rotor-Gene Q Cycler (Qiagen, Valencia, CA, USA) using the SYBR GREEN PCR Master mix (Qiagen). The data were analyzed using the $2^{-\triangle \Delta C T}$ method and amplification was performed for $2 \mathrm{~min}$ at $50^{\circ} \mathrm{C}$ and $10 \mathrm{~min}$ at $95^{\circ} \mathrm{C}$, followed by 40 cycles of $95^{\circ} \mathrm{C}$ for $10 \mathrm{sec}, 60^{\circ} \mathrm{C}$ for $10 \mathrm{sec}$, and $72^{\circ} \mathrm{C}$ for $30 \mathrm{sec}$. For each gene, the relative expression levels were calculated by comparison with a standard curve, following normalization to the expression of the housekeeping gene $\beta$-actin selected as a control.

Animal experiment. The animal experiments were performed, according to the ethical guidelines and were approved by the animal Ethical Committee of The First Hospital of Jilin University (Jilin, China). Thirty female KLF9 knockout mice and age-matched wild-type female mice (C57BL/6J; 14-16 weeks-old) were purchased from the Chinese Academy of Medical Science (Beijing, China). They were anesthetized and subjected to intratracheal instillation of saline or $2.5 \mathrm{mg} / \mathrm{kg}$ BLM sulfate saline solution (Sigma-Aldrich). The mice were euthanized via cervical dislocation 10 days following instillation and the content of 8-oxo-20-deoxyguanosine $(8-\mathrm{OHdG})$, a marker of oxidative DNA damage, was determined in the DNA isolated from the lungs of the treated animals.

Histopathological examination. At 19 days following intratracheal administration of BLM, the mice were euthanized and the lungs were inflated and fixed with $4 \%$ paraformaldehyde. The lungs were surgically resected and embedded in paraffin wax. Lung sections $(5 \mathrm{~mm})$ were cut and stained with hematoxylin and eosin. The stained sections were examined with a light microscope (Eclipse E100-LED; Nikon, Tokyo, Japan).

Statistical analysis. Data are presented as the mean \pm standard deviation of triplicate experiments. Statistical significance was determined by one-way analysis of variance, followed by Tukey's multiple comparison tests. Analyses were conducted using Prism 5.0 (GraphPad Software, San Diego, CA, USA). $\mathrm{P}<0.05$ was considered to indicate a statistically significant difference.

\section{Results}

Effects of BLM treatment. A dose-dependent increase in the production of ROS and cell death were observed in 


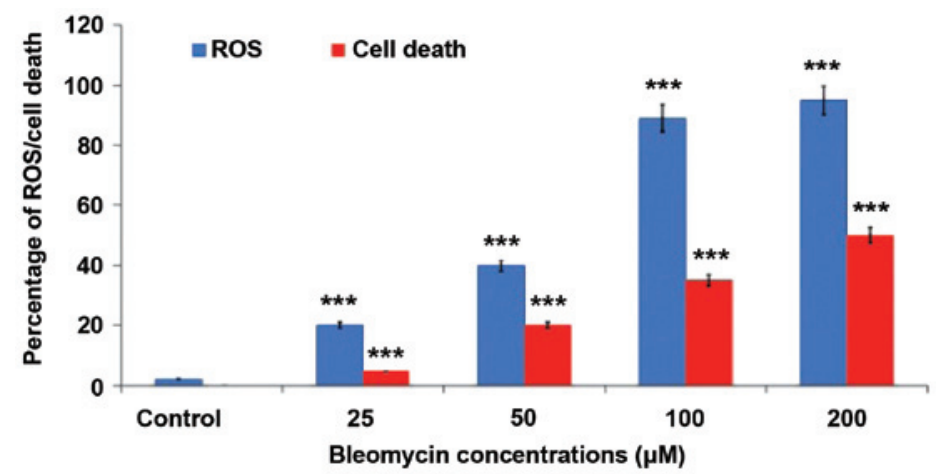

Figure 1. Percentage of ROS production and cell death in HPF cells treated with different concentrations of bleomycin. The percentage of ROS production and the percentage of cell death was calculated by use of a fluorescent probe. The data are expressed as the mean \pm standard deviation ("P $<0.05$, compared with $100 \mu \mathrm{M}$ bleomycin treated cells; ${ }^{* * *} \mathrm{P}<0.001$, compared with $200 \mu \mathrm{M}$ bleomycin treated cells. ROS, reactive oxygen species.

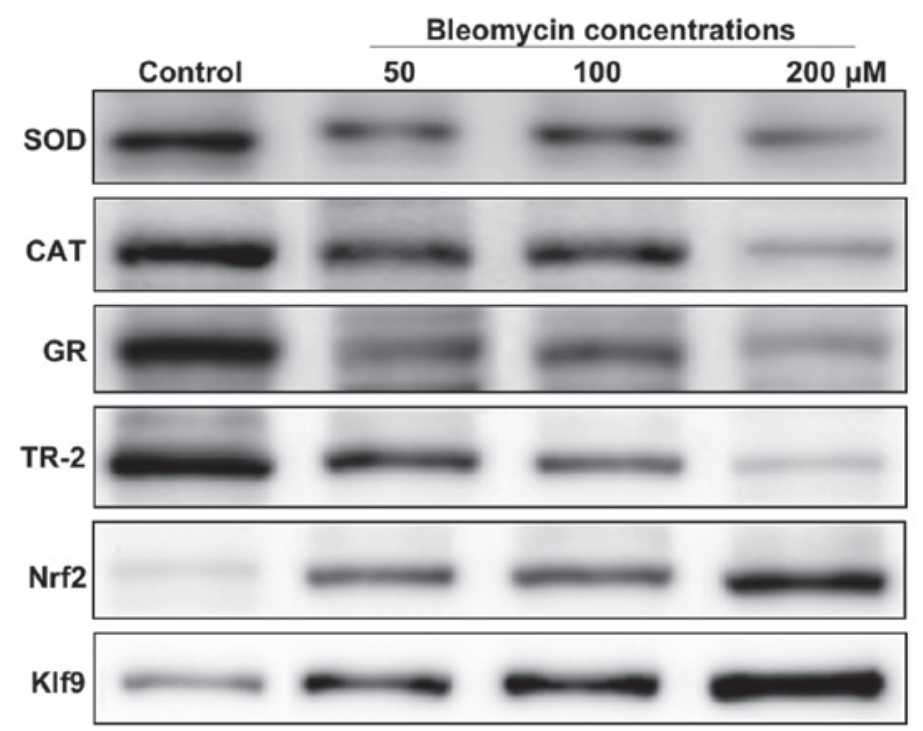

Figure 2. Western blotting was performed to determine the protein expression levels of antioxidant proteins, including SOD, CAT, GR, TR-2, Nrf2 and Klf9, in HPF cells treated with different concentrations $(50,100$ or $200 \mu \mathrm{M})$ of bleomycin. SOD, superoxide dismutase; CAT, catalase; GR, glutathione reductase, TR-2, thioredoxine reductase 2; Nrf2, NF-E2-related transcription factor 2; Klf9, Kruppel-like factor 9.

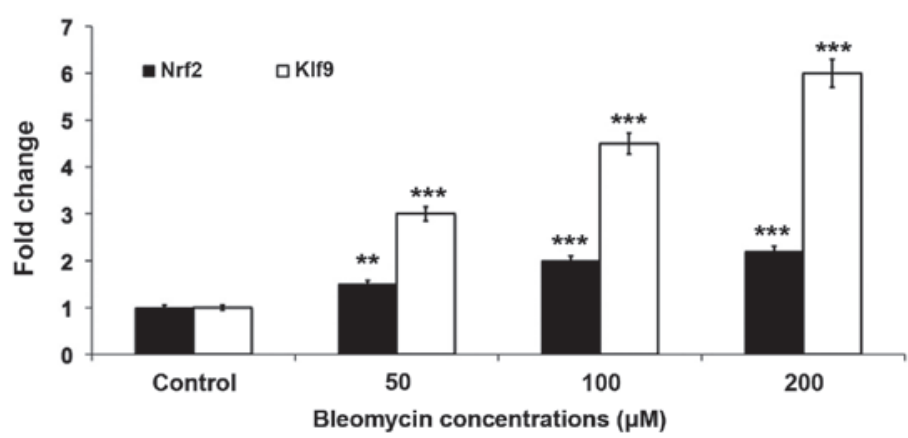

Figure 3. Graphical representation of reverse transcription-quantitative polymerase chain reaction analysis demonstrating the fold increase of the mRNA expression levels of Nrf2 and Klf9 in HPF cells treated with different concentrations (50, 100 or $200 \mu \mathrm{M})$ of bleomycin. The data are expressed as the mean \pm standard deviation $\left({ }^{* *} \mathrm{P}<0.01\right.$, compared with the $100 \mu \mathrm{M}$ BLM treated cells; ${ }^{* * * *} \mathrm{P}<0.001$, compared with the $200 \mu \mathrm{M}$ BLM treated cells). Nrf2, NF-E2-related transcription factor 2; Klf9, Kruppel-like factor 9.

BLM-treated cells compared with the normal untreated cells (Fig. 1). The present study next investigated the levels of the anti-oxidant proteins, CAT, SOD, GR and TR-2, at $24 \mathrm{~h}$ in BLM-treated and control HPFs. The protein expression levels of Nrf2 and Klf9 were markedly increased at concentrations of BLM $>50 \mu \mathrm{M}$ (Fig. 2). However, with the exception of Nrf2 and Klf9, all other antioxidant proteins demonstrated a dose-dependent decrease in the expression levels (Fig. 2). To 
A
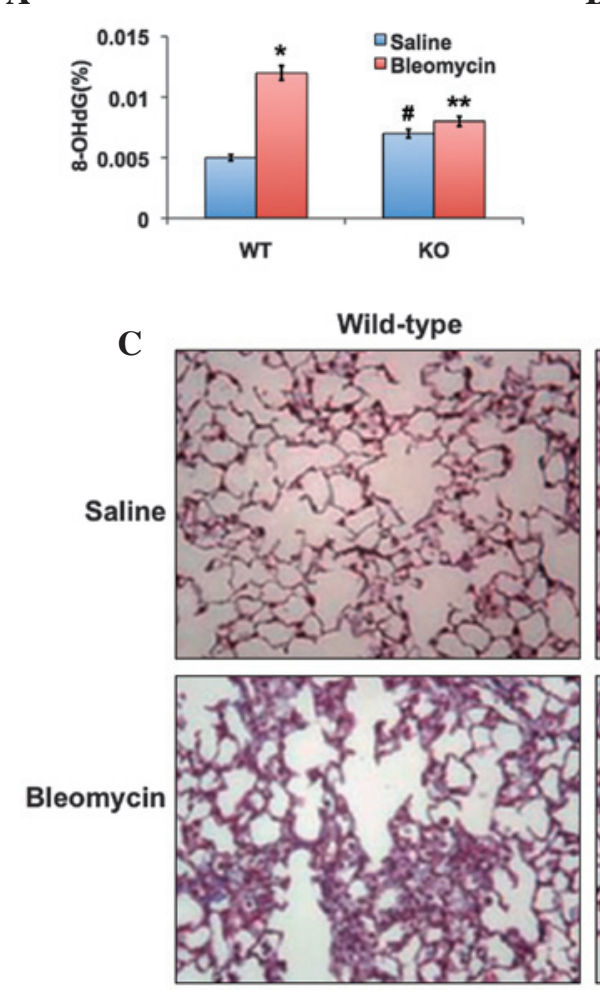

B

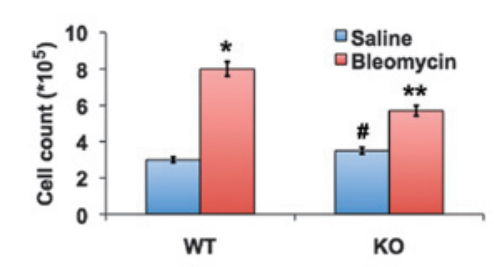

KIf9-knockout

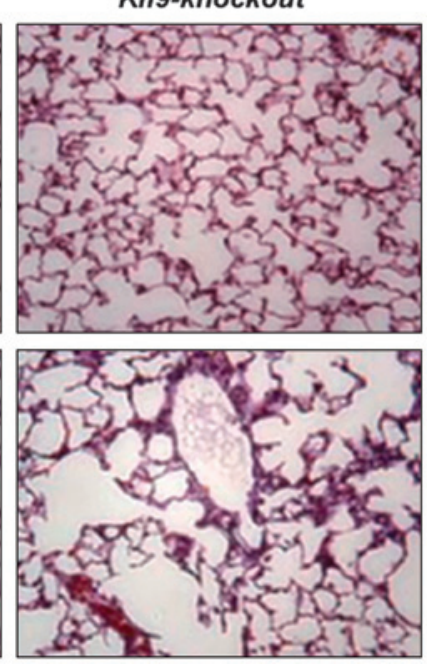

Figure 4. Levels of Klf9 in the lungs of Klf9 knockout mice treated with bleomycin. (A) The levels of oxidative DNA damage were determined by measuring the levels of 8-OHdG in the lung tissues of WT and Klf9 KO mice. Data are presented as the percentage of 8-OHdG of the total DNA analyzed (200 ng). (B) Bronchoalveolar lavage fluid was collected from the animals 19 days following treatment and was analyzed for the total cell counts using a hemocytometer. Data are expressed as the mean \pm standard error of the mean $\left({ }^{*} \mathrm{P}<0.05\right.$, compared with the saline treated WT treated mice; ** $\mathrm{P}<0.05$, compared with the saline treated KO mice; ${ }^{~} \mathrm{P}<0.05$, compared with the bleomycin treated WT mice). (C) Representative histopathological assessment of hematoxylin and eosin stained sections of mice lung from the experimental groups (magnification, x100). 8-OHdG, 8-hydroxy-20-deoxyguanosine; WT, wild-type; KO, knockout; Klf9, Kruppel-like factor 9 .

understand the mechanisms of the Klf9-mediated response to oxidative stress, the gene expression levels of K1f9 and Nrf2 were determined in the BLM treated cells. Similarly, gene expression analysis by RT-qPCR revealed that oxidative stress induced the mRNA expression levels of Nrf2 and Klf9 (Fig. 3). It was revealed that a 4- and 6-fold increase in the gene expression levels of Nrf2 and Klf9, respectively, occurred in the BLM-treated cells.

Effect of Klf9 deficiency on DNA damage and lung fibrosis. BLM treatment induced a statistically significant increase in the levels of $8-\mathrm{OHdG}$, a marker of oxidative DNA damage, in the lungs of wild-type mice $(\mathrm{P}=0.0003)$; however, it revealed no significant effect on the levels of $8-\mathrm{OHdG}$ in the lungs of Klf9 knockout mice (Fig. 4A). A second cohort of saline- or BLM-treated mice were analyzed 19 days following treatment for the accumulation of cells in the bronchoalveolar lavage fluid (BALF) and for the accumulation of collagen, each of which are markers of lung fibrosis. A significant increase in the number of total BALF cells was observed in all animals treated with BLM (Fig. 4B). However, this increase was significantly lower in the Klf9 knockout mice compared with the wild-type counterparts $(\mathrm{P}<0.05)$. Fibrosis was observed in the histological lung sections stained with hematoxylin and eosin (Fig. 4C). No sign of fibrosis was detected in the wild-type or Klf9 knockout mice treated with saline solution. Treatment with BLM led to pulmonary fibrosis in the wild-type and knockout animals, although the degree of fibrosis was significantly higher in the wild-type mice compared with the knockout counterparts $(\mathrm{P}=0.038)$. Therefore, Klf9 deficiency provided resistance to BLM-induced oxidative stress and pulmonary fibrosis in mice.

\section{Discussion}

Due to the wide range of toxicities (pulmonary toxicity) associated with BLM (antineoplastic drug), clinical use is yet to be effective. Although several mechanisms have been hypothesized to explain BLM-induced pulmonary toxicity, there is clear evidence that ROS contribute to the pathogenesis of BLM-induced lung injury through several pathways (15-17). Pulmonary fibrosis is a chronic multifactorial disease, where oxidative stress is important in disease progression (18). In the present study, the transcription factor, Klf9, was assessed as a regulator of intracellular ROS in BLM-induced pulmonary toxicity. Klf9 is a ubiquitously expressed protein with a relatively understudied function. A significant, concentration-dependent increase in the total ROS was observed in the present study, indicating an increase in oxidative stress in response to treatment with BLM (Fig. 1). These data are in agreement with other previous studies, which have reported an increase in the production of ROS upon treatment with BLM $(2,19)$. Antioxidant enzymes are involved in the detoxification or scavenging of oxidative free radicals in 
the cells. These results suggested that upon treatment with BLM, there is a significant reduction in the major antioxidant proteins, CAT, SOD, GR and TR-2, which are hypothesized to be an effective defense system in the lung. This indicated the excess generation of ROS (Fig. 2). This data is consistent with previous studies indicating that a decrease in antioxidant activity following BLM treatment indicated the formation of ROS, which, in turn, caused further damage to lungs (20-22). However, an increase in the expression levels of Nrf2 (23) and Klf9 upon treatment with BLM has also been reported (24).

The chemotherapeutic agent, BLM, has been demonstrated to cause oxidative lung injuries in mice, and BLM-treated mice are considered to be a classical model of idiopathic pulmonary fibrosis (25). Currently, only a few genes involved in the control of ROS have been implicated in the pathogenesis of pulmonary fibrosis in mouse knockout models (18), including Nrf2 as a sole transcription factor among these genes (12). Furthermore, Nrf2 was activated and its targets, including Nqo1, were upregulated in mice treated with BLM (23). Therefore, the functional involvement of Klf9, and potentially its targets, in the pathogenesis of pulmonary fibrosis uncovers a regulatory network of this disease and may offer targets and/or prognostic markers. The present investigation using Klf9 knockout mice clearly elucidated the reduced risk of ROS formation in lungs of BLM-treated mice. Since Klf9 depletion also suppressed oxidative stress, Klf9 downregulation may promote cancer progression and, potentially, resistance to the treatment. In conclusion, the data suggested that Klf9 is upregulated by Nrf 2 under conditions of excessive oxidative stress, therefore, suggesting unique functions and modalities of the action of Nrf2 in the cell. These results are consistent with studies regarding the prognostic value of the expression of K1f9 in cancer and specifically Nrf2-regulated expression of Klf9. Investigations focusing on the involvement of Nrf2 and K1f9 are important for determining future therapeutic targets for BLM-induced pulmonary toxicity.

\section{References}

1. Karam H, Hurbain-Kosmath I and Housset B: Antioxidant activity in alveolar epithelial type 2 cells of rats during the development of bleomycin injury. Cell Biol Toxicol 14: 13-22, 1998.

2. Patel RB, Kotha SR, Sauers LA, Malireddy S, Gurney TO, Gupta NN, Elton TS, Magalang UJ, Marsh CB, Haley BE, et al: Thiol-redox antioxidants protect against lung vascular endothelial cytoskeletal alterations caused by pulmonary fibrosis inducer, bleomycin: Comparison between classical thiol-protectant, $\mathrm{N}$-acetyl-L-cysteine and novel thiol antioxidant, N,N'-bis-2-mercaptoethyl isophthalamide. Toxicol Mech Methods 22: 383-396, 2012.

3. Lazo JS and Chabner BA: Cancer chemotherapy and biotherapy: principles and practice. In: Chabner BA, Longo DL (eds) Philadelphia: Lippincott-Raven, 379-394, 1996.

4. Hecht SM: Bleomycin: New perspectives on the mechanism of action. J Nat Prod 63: 158-168, 2000.
5. Chandler DB: Possible mechanisms of bleomycin-induced fibrosis. Clin Chest Med 11: 21-30, 1990.

6. Keane MP, Belperio JA, Arenberg DA, Burdick MD, Xu ZJ, Xue YY and Strieter RM: IFN-gamma-inducible protein-10 attenuates bleomycin-induced pulmonary fibrosis via inhibition of angiogenesis. J Immunol 163: 5686-5692, 1999.

7. Finkel $\mathrm{T}$ and Holbrook NJ: Oxidants, oxidative stress and the biology of ageing. Nature 408: 239-247, 2000.

8. Cross CE, Warren D, Gerriets JE, Wilson DW, Halliwell B and Last JA: Deferoxamine injection does not affect bleomycin induced lung fibrosis in rats. J Lab Clin Med 106: 433-438, 1985.

9. Itoh K, Wakabayashi N, Katoh Y, Ishii T, Igarashi K, Engel JD and Yamamoto M: Keap1 represses nuclear activation of antioxidant responsive elements by Nrf2 through binding to the amino-terminal Neh2 domain. Genes Dev 13: 76-86, 1999.

10. Wasserman WW and Fahl WE: Functional antioxidant responsive elements. Proc Natl Acad Sci USA 94: 5361-5366, 1997.

11. Itoh K, Chiba T, Takahashi S, Ishii T, Igarashi K, Katoh Y, Oyake T, Hayashi N, Satoh K, Hatayama I, et al: An Nrf2/small Maf heterodimer mediates the induction of phase II detoxifying enzyme genes through antioxidant response elements. Biochem Biophys Res Commun 236: 313-322, 1997.

12. Kikuchi N, Ishii Y, Morishima Y, Yageta Y, Haraguchi N, Itoh K, Yamamoto $\mathrm{M}$ and Hizawa N: Nrf2 protects against pulmonary fibrosis by regulating the lung oxidant level and Th1/Th2 balance. Respir Res 11: 31, 2010.

13. Mannava S, Zhuang D, Nair JR, Bansal R, Wawrzyniak JA, Zucker SN, Fink EE, Moparthy KC, Hu Q, Liu S, et al: KLF9 is a novel transcriptional regulator of bortezomib- and LBH589-induced apoptosis in multiple myeloma cells. Blood 119: 1450-1458, 2012.

14. Bradford MM: A rapid and sensitive method for the quantitation of microgram quantities of protein utilizing the principle of protein-dye binding. Anal Biochem 72: 248-254, 1976.

15. Serrano-Mollar A, Closa D, Prats N, Blesa S, Martinez-Losa M, Cortijo J, Estrela JM, Morcillo EJ and Bulbena O: In vivo antioxidant treatment protects against bleomycin-induced lung damage in rats. Br J Pharmacol 138: 1037-1048, 2003.

16. Mastruzzo C, Crimi N and Vancheri C: Role of oxidative stress in pulmonary fi brosis. Monaldi Arch Chest Dis 57: 173-176, 2002.

17. Mata M, Ruiz A, Cerda M, Martinez-Losa M, Cortijo J, Santangelo F, Serrano-Mollar A, Llombart-Bosch A and Morcillo EJ: Oral N-acetylcysteine reduces bleomycininduced lung damage and mucin Muc5ac expression in rats. Eur Respir J 22: 900-905, 2003.

18. Cheresh P, Kim SJ, Tulasiram S and Kamp DW: Oxidative stress and pulmonary fibrosis. Biochim Biophys Acta 1832: 1028-1040, 2013.

19. Sayed-Ahmed MM, Mansour HH, Gharib OA and Hafez HF: Acetyl-L-carnitine modulates bleomycin-induced oxidative stress and energy depletion in lung tissues. J Egypt Natl Canc Inst 16: 237-243, 2004.

20. Ali EN and Mansour SZ: Boswellic acids extract attenuates pulmonary fi brosis induced by bleomycin and oxidative stress from gamma irradiation in rats. Chin Med 6: 36, 2011.

21. Iraz M, Erdogan H, Kotuk M, Yagmurca M, Kilic T, Ermis H, Fadillioğlu E and Yildirim Z: Ginkgo biloba inhibits bleomycin-induced lung fi brosis in rats. Pharmacol Res 53: 310-316, 2006.

22. Erdogan H, Fadillioglu E, Kotuk M, Iraz M, Tasdemir S, Oztas Y and Yildirim Z: Effects of Ginkgo biloba on plasma oxidant injury induced by bleomycin in rats. Toxicol Ind Health 22: 47-52, 2006.

23. Cho HY, Reddy SP, Yamamoto M and Kleeberger SR: The transcription factor NRF2 protects against pulmonary fibrosis. FASEB J 18: 1258-1260, 2004.

24. Bieker JJ: Krüppel-like factors: Three fingers in many pies. J Biol Chem 276: 34355-34358, 2001.

25. Mouratis MA and Aidinis V: Modeling pulmonary fibrosis with bleomycin. Curr Opin Pulm Med 17: 355-361, 2011. 